SCIENTIFIC LETTER

\title{
Low prevalence of cardiac troponin T mutations in a Greek hypertrophic cardiomyopathy cohort
}

\author{
A Miliou, A Anastasakis, L G D'Cruz, A Theopistou, A Rigopoulos, I Rizos, S Stamatelopoulos, \\ P Toutouzas, C Stefanadis
}

Heart 2005;91:966-967. doi: 10.1136/hrt.2003.029819

S ince the initial description of hypertrophic cardiomyopathy (HCM), sudden cardiac death (SCD) occurring in young and usually asymptomatic patients has received substantial attention in the literature as the most devastating consequence of the disease. Present data from non-referral centres show a benign course of the disease with a subgroup of high risk patients. ${ }^{1}$

Molecular genetics have reconfirmed familial HCM as being an autosomal dominant disease with hundreds of mutations in 10 genes encoding sarcomeric proteins. ${ }^{1}$ Cardiac troponin T (TNNT2) gene mutations have been reported to be consistently associated with a malignant clinical prognosis and the prevalence has been reported to be in the region of $15-20 \% .^{2}$ Recent epidemiological studies in unselected HCM populations lower this percentage dramatically. ${ }^{3}$ Clinical expression and prognosis of HCM in Greece seems to have a benign course and a moderate morphologic expression. ${ }^{4}$

So far, the prevalence and type of mutations for any HCM associated gene has not been reported in Greece. Screening the Greek HCM probands for TNNT2 mutations is the first step to assess the occurrence and overall prevalence of the supposed more aggressive and malignant subset of HCM mutations.

\section{METHODS}

One hundred and forty three unrelated families of Greek origin ( 118 men, mean age $47 \pm 16$ years) participated. The disease was familial in 81 cases $(56.6 \%)$ and 22 patients (15.3\%) had a family history of SCD. ${ }^{4}$

All patients fulfilled conventional criteria for familial $\mathrm{HCM}^{1}$ and had a maximal left ventricular wall thickness of 19.1 (4.8) $\mathrm{mm}$. Referral was based on disease diagnosis according to World Health Organization criteria. ${ }^{1}$ Baseline evaluation included clinical examination, 12 lead ECG, echocardiography, and 24 hour ambulatory ECG monitoring. ${ }^{5}$ Follow up was performed at six monthly intervals.

Genomic DNA was isolated from the blood samples of probands, relatives, and 100 healthy Greek subjects, using the BACC2 Nucleon kit (Amersham, UK).

Mutation analysis was performed by means of polymerase chain reaction (PCR), single strand conformation polymorphism (SSCP), and sequencing. Exonic and adjacent intronic sequences were amplified by use of oligonucleotide primers ${ }^{1}$ and PCR (Eppendorf Mastercycler Gradient: Brinkmann Instruments). The PCR products were sequenced after the identification of altered SSCP patterns at two different temperatures (Consort, Turnhout) and by use of an automated sequencer directly after PCR. All products were directly sequenced, bidirectionally (MWG Biotech, Germany). Each mutation was confirmed by a new PCR on a fresh blood sample and re-evaluated by an independent researcher with no prior knowledge of results.

\section{RESULTS}

Mutation screening of the 143 Greek probands revealed four probands with three different mutations in the TNNT2 gene. The prevalence of TNNT2 mutations in our cohort was found to be $2.8 \%$. All three mutations were found in the last exon. All mutations identified were missense and in regions where the amino acid sequence indicated high conservation throughout evolution. None of the mutations were detectable in any of the 100 healthy controls.

Sequencing revealed an Arg278Cys mutation. Proband A (AIII: 5) was diagnosed with HCM at young age and died suddenly at the age of 16 years. There was no family history of SCD. Proband B (B IV: 1) was diagnosed at the age of 32 years with HCM. SCDs have been experienced in the family at advanced age. One relative (B III: 11) with typical disease was found to be a carrier and three others were normal carriers.

Mutation screening for proband C (C III: 1) revealed an Arg278Pro mutation. Proband C was diagnosed with typical HCM at the age of 18 years. His father (C II: 1) was also found to be an affected carrier. There is no family history of SCD.

A recently reported $\operatorname{Arg} 286$ Cys $^{1}$ substitution was found for proband D (D III: 1), exhibiting a typical form of HCM diagnosed at the age of 26 years. One more family member (D IV: 1), 26 years old, was detected as a carrier with no echocardiographic evidence but with ECG criteria for the subclinical form of the disease in relatives. ${ }^{1}$ There is no family history of SCD (fig 1).

Three polymorphisms were revealed, all in exonic sequences. A new silent variant, in exon 8 (cl2739G $>$ A), a second one in exon 9 (t13150C $>$ T), and a third, rare, variant in exon 14 resulting in Ile253Arg substitution (AAG>AGG). ${ }^{2}$

\section{DISCUSSION}

Unrelated Greek HCM patients were assessed for the presence of cardiac TNNT2 mutations. Three mutations were found in four different families. The prevalence of TNNT2 mutations in the Greek HCM cohort was found to be $2.8 \%$. All mutations were found to be in the last exon of the gene.

Reports from other groups are in agreement with such low frequencies. ${ }^{3}$ Previous studies have reported the occurrence of TNNT2 mutations to be approximately $15 \%{ }^{2}$ The discrepancy in the apparent prevalence of TNNT2 gene mutations between previous and current studies is probably because of a more stringent patient selection criteria compared to the selection bias normally encountered in tertiary referral centres. The cohort of this study fulfils the criteria of a representative, unselective, Greek HCM population. ${ }^{4}$ Clustering of all mutations at exon 16 for the Greek cohort may represent a differing mutational pool from the populations studied already.

Abbreviations: TNNT2, cardiac troponin T; HCM, hypertrophic cardiomyopathy; PCR, polymerase chain reaction; SSCP, single strand conformation polymorphism; SCD, sudden cardiac death 


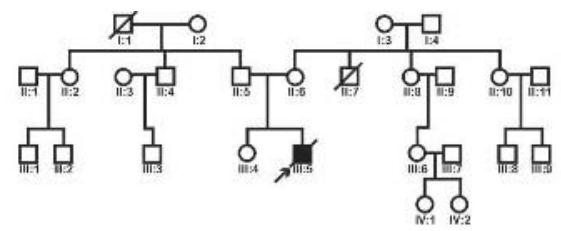

Family A
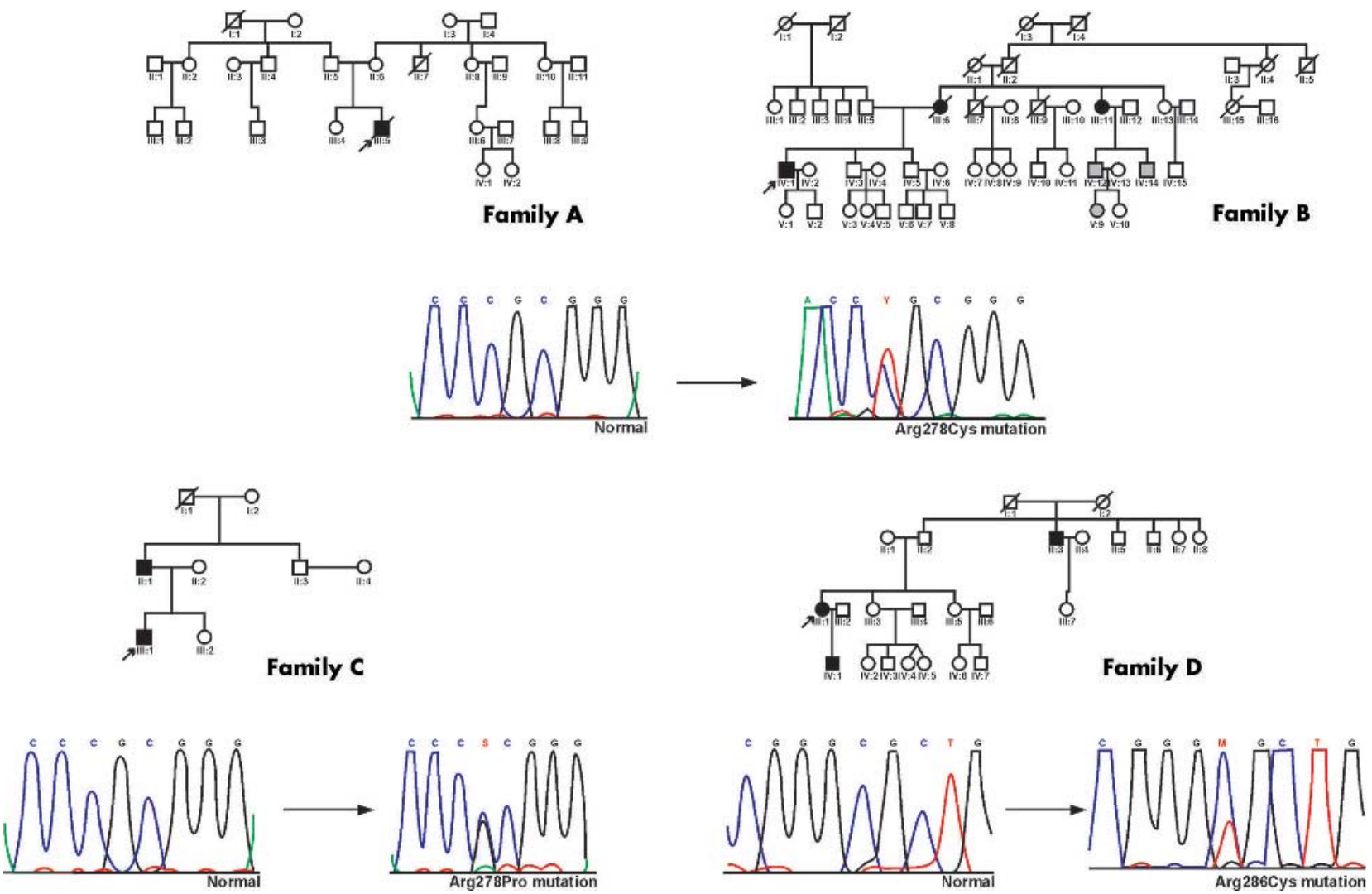

Figure 1 Pedigrees of families A, B, C, and D and their respective mutations (families A and B: Arg278Cys mutation; family C: Arg278Pro mutation; and family D: Arg286Cys mutation).

Two unrelated probands were found to be carriers of the mutation Arg278Cys. Survival characteristics and presence of the disease were quite distinct in the two cases but in agreement with international bibliography. ${ }^{125}$ Family history of SCD in the young was the exception rather than the rule in our families (fig 1).

So far approximately 27 mutations have been identified for the TNNT2 gene, mostly associated with the more malignant course of the disease. A clear phenotype/genotype correlation has not yet been deduced, ${ }^{1}$ indicating the need for a strategy that will take into account populations of common geographical areas.

In Greece, genetic screening for TNNT2 mutations, focusing on exon 16, may be a rational approach to widespread genetic screening in HCM. This would provide a relatively inexpensive first line genetic analysis of HCM, especially where clinical criteria fail because of incomplete or non-penetrance.

In general, for Greece, TNNT2 gene mutation frequency is low, for its homogeneous, well characterised, unselected HCM population. The clinical expression seems variable and needs further investigation.

\section{Authors' affiliations}

A Miliou, A Anastasakis, A Theopistou, A Rigopoulos*, I Rizos*, P Toutouzas, C Stefanadis, Centre for the Study of Inherited Cardiovascular Diseases, Cardiology Department, Hippokrateion Hospital, Medical School, University of Athens, Athens, Greece
L G D'Cruz†, Department of Cardiovascular Medicine, St George's Hospital, University of London, London, UK

S Stamatelopoulos, Department of Cardiology, Errikos Dinan Hospital, Athens, Greece

*Now at Department of Cardiology, Attikon Hospital, Athens, Greece †Also at Division of Protein Structure, MRC National Institute of Medical Research, Mill Hill, London, UK

Correspondence to: Mrs Antigoni Miliou, 4 Trias Street, N Erithrea, 146 71, Athens, Greece; a_miliou@hotmail.com

Accepted 15 July 2004

\section{REFERENCES}

1 American College of Cardiology, European Society of Cardiology. ACC/ESC Clinical expert consensus document on hypertrophic cardiomyopathy. Eur Heart J 2003;24:1965-91

2 Watkins H, McKenna WJ, Thierfelder L, et al. Mutations in the genes for cardiac troponin T and $\alpha$ tropomyosin in hypertrophic cardiomyopathy. N Engl J Med 1995;332:1058-64.

3 Torricelli F, Girolami F, Olivotto I, et al. Prevalence and clinical profile of troponin T mutations among patients with hypertrophic cardiomyopathy in Tuscany. Am J Cardiol 2003;92:1358-62.

4 Kyriakidis M, Triposkiadis F, Anastasakis A, et al. Hypertrophic cardiomyopathy in Greece: clinical course and outcome. Chest 1998;114:1091-6.

5 Elliott PM, D Cruz LG, McKenna WJ. Late onset hypertrophic cardiomyopathy caused by a mutation in the cardiac troponin T gene. N Engl J Med 1999;341:1855-6. 